\title{
Two-phase flows involving capillary barriers in heterogeneous porous media
}

\author{
CLÉMENT CANCÈS \\ ENS Cachan Bretagne, IRMAR, UEB, av. Robert Schuman, 35170 Bruz, France \\ E-mail: clement.cances@bretagne.ens-cachan.fr \\ THIERRY GALLOUËT \\ Université de Provence, 39, rue F. Joliot Curie, 13453 Marseille Cedex 13, France \\ E-mail: gallouet@cmi.univ-mrs.fr \\ Alessio PorRetTA \\ Università di Roma Tor Vergata, Via della Ricerca Scientifica 1, 00133 Roma, Italy \\ E-mail: porretta@mat.uniroma2.it
}

[Received 11 November 2007 and in revised form 2 September 2008]

\begin{abstract}
We consider a simplified model of a two-phase flow through a heterogeneous porous medium, in which convection is neglected. This leads to a nonlinear degenerate parabolic problem in a domain divided into an arbitrary finite number of homogeneous porous media. We introduce a new way to connect capillary pressures on the interfaces between the homogeneous domains, which leads to a general notion of solution. We then compare this notion of solution with an existing one, showing that it allows one to deal with a larger class of problems. We prove the existence of such a solution in a general case, and the existence and uniqueness of a regular solution in the one-dimensional case for initial data regular enough.
\end{abstract}

Keywords: Flows in porous media; capillarity; nonlinear PDE of parabolic type.

\section{Presentation of the problem}

Models of immiscible two-phase flows are widely used in petroleum engineering, particularly in basin modelling, whose aim can be the prediction of the migration of hydrocarbon components at geological time scale in a sedimentary basin.

The heterogeneousness of the porous medium leads to the phenomena of oil-trapping and oil-expulsion, which is modelled with discontinuous capillary pressures between the different geological layers.

The physical and mathematical models can be found in [4, 5, 12, 13, 14]. The phenomenon of capillary trapping has been considered only in simplified cases (see [6]), and several numerical methods have been developed (see e.g. [16, 15]).

The aim of this paper is to introduce a new notion of weak solution, which allows us to deal with more general cases than those treated in [15], while it is equivalent to the notion of weak solution introduced in [15] in the already treated cases. We will consider a simplified model $\mathcal{P}$ ) (page 242), in which convection is neglected.

We then give a uniqueness result in the one-dimensional case which is inspired from the result in [6] and extends it to more general situations, by requiring weaker assumptions on the solutions and applying to a larger class of initial data.

We have to make some assumptions on the heterogeneous porous medium: 
ASSUMPTIONS 1 (Geometrical assumptions)

1. The heterogeneous porous medium is represented by a polygonal bounded connected domain $\Omega \subset \mathbb{R}^{d}$ with meas $\mathbb{R}^{d}(\Omega)>0$, where meas $\mathbb{R}^{n}$ is the Lebesgue measure on $\mathbb{R}^{n}$.

2. There exist a finite number $N$ of polygonal connected subdomains $\left(\Omega_{i}\right)_{1 \leqslant i \leqslant N}$ of $\Omega$ such that:

(a) for all $i \in \llbracket 1, N \rrbracket$, meas $\mathbb{R}^{d}\left(\Omega_{i}\right)>0$,

(b) $\bigcup_{i=1}^{N} \bar{\Omega}_{i}=\bar{\Omega}$,

(c) for $(i, j) \in \llbracket 1, N \rrbracket^{2}$ with $i \neq j, \Omega_{i} \cap \Omega_{j}=\emptyset$.

Each $\Omega_{i}$ represents a homogeneous porous medium. For all $(i, j) \in \llbracket 1, N \rrbracket^{2}$, one denotes by $\Gamma_{i, j} \subset \Omega$ the interface between the geological layers $\Omega_{i}$ and $\Omega_{j}$, defined by $\bar{\Gamma}_{i j}=\partial \Omega_{i} \cap \partial \Omega_{j}$.

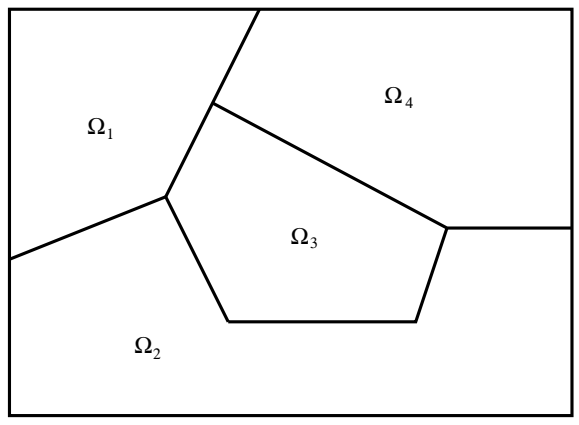

FIG. 1. An example of the domain $\Omega$.

We consider an incompressible and immiscible oil-water flow through $\Omega$, and thus through each $\Omega_{i}$. By Darcy's law, the conservation of oil and water phases is given for all $\left.(x, t) \in \Omega_{i} \times\right] 0, T$ [ by the equations

$$
\left\{\begin{array}{l}
\phi_{i} \partial_{t} u_{i}(x, t)-\nabla \cdot\left(\eta_{o, i}\left(u_{i}(x, t)\right)\left(\nabla p_{o, i}(x, t)-\rho_{o} \mathbf{g}\right)\right)=0, \\
-\phi_{i} \partial_{t} u_{i}(x, t)-\nabla \cdot\left(\eta_{w, i}\left(u_{i}(x, t)\right)\left(\nabla p_{w, i}(x, t)-\rho_{w} \mathbf{g}\right)\right)=0, \\
p_{o, i}(x, t)-p_{w, i}(x, t)=\pi_{i}\left(u_{i}(x, t)\right),
\end{array}\right.
$$

where $u_{i} \in[0,1]$ is the oil saturation in $\Omega_{i}$ (and therefore $1-u_{i}$ the water saturation), $\phi_{i} \in$ ]0, 1[ is the porosity of $\Omega_{i}$, which is supposed to be constant in each $\Omega_{i}$ for the sake of simplicity, $\pi_{i}\left(u_{i}(x, t)\right)$ is the capillary pressure, and $\mathbf{g}$ is the gravity acceleration. The indices $o$ and $w$ stand for the oil and the water phases respectively. Thus, for $\sigma=o, w, p_{\sigma, i}$ is the pressure, $\eta_{\sigma, i}$ is the mobility, and $\rho_{\sigma}$ is the density of the phase $\sigma$.

We now have to make assumptions on the data to specify the transmission conditions through the interfaces $\Gamma_{i, j}$ :

ASSUMPTIONS 2 (Assumptions on the data)

1. For all $i \in \llbracket 1, N \rrbracket, \pi_{i} \in C^{1}([0,1], \mathbb{R})$, with $\pi_{i}^{\prime}(x)>0$ for $\left.x \in\right] 0,1[$.

2. For all $i \in \llbracket 1, N \rrbracket, \eta_{o, i} \in C\left([0,1], \mathbb{R}_{+}\right)$is an increasing function with $\eta_{o, i}(0)=0$.

3. For all $i \in \llbracket 1, N \rrbracket, \eta_{w, i} \in C\left([0,1], \mathbb{R}_{+}\right)$is a decreasing function with $\eta_{w, i}(1)=0$.

4. The initial data $u_{0}$ belongs to $L^{\infty}(\Omega), 0 \leqslant u_{0} \leqslant 1$. 


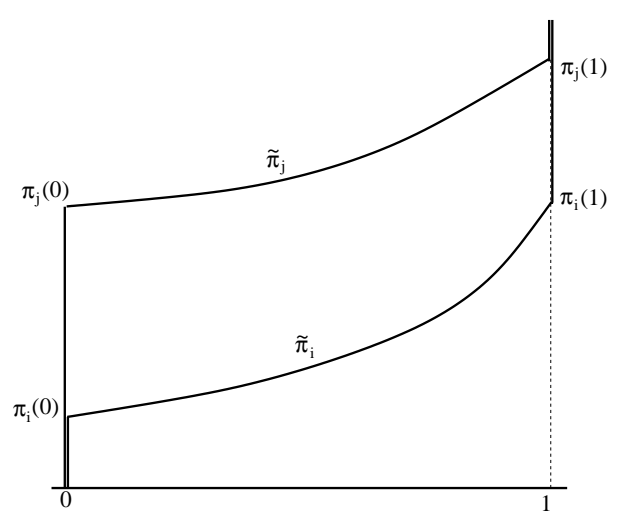

FIG. 2. Graphs for the capillary pressures. by

We set $\alpha_{i}=\lim _{s \rightarrow 0} \pi_{i}(s)$ and $\beta_{i}=\lim _{s \rightarrow 1} \pi_{i}(s)$. We can now define the monotonic graphs $\tilde{\pi}_{i}$

$$
\tilde{\pi}_{i}(s)= \begin{cases}\pi_{i}(s) & \text { if } s \in] 0,1[, \\ ]-\infty, \alpha_{i}\right] & \text { if } s=0, \\ {\left[\beta_{i},+\infty[\right.} & \text { if } s=1 .\end{cases}
$$

As shown in [15], the following conditions must be satisfied on the traces of $u_{i}, p_{\sigma, i}$ and $\nabla p_{\sigma, i}$ on $\left.\Gamma_{i, j} \times\right] 0, T\left[\right.$, still denoted respectively $u_{i}, p_{\sigma, i}$ and $\nabla p_{\sigma, i}$ (see [5]):

1. For any $\sigma=o, w$, and $(i, j) \in \llbracket 1, N \rrbracket^{2}$ such that $\Gamma_{i, j} \neq \emptyset$, the flux of the phase $\sigma$ through $\Gamma_{i, j}$ must be continuous:

$$
\eta_{\sigma, i}\left(u_{i}\right)\left(\nabla p_{\sigma, i}-\rho_{\sigma} \mathbf{g}\right) \cdot \mathbf{n}_{i}+\eta_{\sigma, j}\left(u_{j}\right)\left(\nabla p_{\sigma, j}-\rho_{\sigma} \mathbf{g}\right) \cdot \mathbf{n}_{j}=0,
$$

where $\mathbf{n}_{i}$ denotes the outward normal to $\Gamma_{i, j}$ pointing to $\Omega_{i}$.

2. For any $\sigma=o, w$, and $(i, j) \in \llbracket 1, N \rrbracket^{2}$ such that $\Gamma_{i, j} \neq \emptyset$, either $p_{\sigma}$ is continuous or $\eta_{\sigma}=0$. Since the saturation is itself discontinuous across $\Gamma_{i, j}$, one must express the mobility at the upstream side of the interface. This gives

$$
\eta_{\sigma, i}\left(u_{i}\right)\left(p_{\sigma, i}-p_{\sigma, j}\right)^{+}-\eta_{\sigma, j}\left(u_{j}\right)\left(p_{\sigma, j}-p_{\sigma, i}\right)^{+}=0 .
$$

The conditions (4) have direct consequences for the behaviour of the capillary pressures on both sides of $\Gamma_{i, j}$. Indeed, if $0<u_{i}, u_{j}<1$, then the partial pressures $p_{o}$ and $p_{w}$ both have to be continuous, and so we have the continuity of the capillary pressures $\pi_{i}\left(u_{i}\right)=\pi_{j}\left(u_{j}\right)$. If $u_{i}=0$ and $0<u_{j}<1$, then $p_{o, i} \geqslant p_{o, j}$ and $p_{w, i}=p_{w, j}$, thus $\pi_{j}\left(u_{j}\right) \leqslant \pi_{i}(0)$. The same way, $u_{i}=1$ and $0<u_{j}<1$ implies $\pi_{j}\left(u_{j}\right) \geqslant \pi_{i}(1)$. If $u_{i}=0, u_{j}=1$, then $p_{o, i} \geqslant p_{o, j}$ and $p_{w, i} \leqslant p_{w, j}$, so $\pi_{i}(0) \geqslant \pi_{j}(1)$. Checking that the definition of the graphs $\tilde{\pi}_{i}$ and $\tilde{\pi}_{j}$ implies $\tilde{\pi}_{i}(0) \cap \tilde{\pi}_{j}(0) \neq \emptyset$, $\tilde{\pi}_{i}(1) \cap \tilde{\pi}_{j}(1) \neq \emptyset$, we can claim that (4) leads to

$$
\tilde{\pi}_{i}\left(u_{i}\right) \cap \tilde{\pi}_{j}\left(u_{j}\right) \neq \emptyset .
$$

We introduce the global pressure in $\Omega_{i}$,

$$
\bar{p}_{i}(x, t)=p_{w, i}(x, t)+\int_{0}^{u_{i}(x, t)} \frac{\eta_{o, i}(a)}{\eta_{o, i}(a)+\eta_{w, i}(a)} \pi_{i}^{\prime}(a) \mathrm{d} a
$$


(see e.g. [3] or [12]), and the global mobility in $\Omega_{i}$,

$$
\lambda_{i}\left(u_{i}(x, t)\right)=\frac{\eta_{o, i}\left(u_{i}(x, t)\right) \eta_{w, i}\left(u_{i}(x, t)\right)}{\eta_{o, i}\left(u_{i}(x, t)\right)+\eta_{w, i}\left(u_{i}(x, t)\right)},
$$

which satisfies $\lambda_{i}(0)=\lambda_{i}(1)=0$, and $\lambda_{i}(s)>0$ for $0<s<1$. Taking into account (6) and (7) in (1), and adding the conservation laws, leads to, for $\left.(x, t) \in \Omega_{i} \times\right] 0, T[$,

$$
\left\{\begin{array}{l}
\phi_{i} \partial_{t} u_{i}(x, t)-\nabla \cdot\left(\eta_{o, i}\left(u_{i}(x, t)\right)\left(\nabla \bar{p}_{i}(x, t)-\rho_{o} \mathbf{g}\right)-\lambda_{i}\left(u_{i}(x, t)\right) \nabla \pi_{i}\left(u_{i}(x, t)\right)\right)=0, \\
-\nabla \cdot\left(\sum_{\sigma=o, w} \eta_{\sigma, i}\left(u_{i}(x, t)\right)\left(\nabla \bar{p}_{i}(x, t)-\rho_{\sigma} \mathbf{g}\right)\right)=0 .
\end{array}\right.
$$

We neglect the convective effects, so that we focus on the mathematical modelling of flows with discontinuous capillary pressures, which seems necessary to explain the phenomena of oil trapping. This simplification will allow us to neglect the coupling with the second equation of (8). Then we get the simple degenerate parabolic equation in $\left.\Omega_{i} \times\right] 0, T[$ :

$$
\left.\phi_{i} \partial_{t} u_{i}(x, t)-\nabla \cdot\left(\lambda_{i}\left(u_{i}(x, t)\right) \nabla \pi_{i}\left(u_{i}(x, t)\right)\right)=0 \quad \text { in } \Omega_{i} \times\right] 0, T[.
$$

In this simplified framework, the transmission condition (3) on the fluxes through $\Gamma_{i, j}$ can be rewritten

$$
\left.\lambda_{i}\left(u_{i}(x, t)\right) \nabla\left(\pi_{i}\left(u_{i}(x, t)\right)\right) \cdot \mathbf{n}_{i}+\lambda_{j}\left(u_{j}(x, t)\right) \nabla\left(\pi_{j}\left(u_{j}(x, t)\right)\right) \cdot \mathbf{n}_{j}=0 \quad \text { on } \Gamma_{i, j} \times\right] 0, T[.
$$

We suppose furthermore that $u_{i}(x, 0)=u_{0}(x)$ for $x \in \Omega_{i}$. In the remainder of this paper, we assume a homogeneous Neumann boundary condition. The existence of a weak solution proven in Section 3 can be extended to the case of nonhomogeneous Dirichlet conditions. Nevertheless, homogeneous Neumann boundary conditions are needed to prove Theorem 4.1, and thus to prove the final Theorem 5.4

Taking into account the equations (5), (9), (10), the boundary condition, and the initial condition, we can write the problem we aim to solve this way: for all $i \in \llbracket 1, N \rrbracket$, and all $j \in \llbracket 1, N \rrbracket$ such that $\Gamma_{i, j} \neq \emptyset$,

$$
\begin{cases}\phi_{i} \partial_{t} u_{i}-\nabla \cdot\left(\lambda_{i}\left(u_{i}\right) \nabla \pi_{i}\left(u_{i}\right)\right)=0 & \text { in } \left.\Omega_{i} \times\right] 0, T[, \\ \tilde{\pi}_{i}\left(u_{i}\right) \cap \tilde{\pi}_{j}\left(u_{j}\right) \neq \varnothing & \text { on } \left.\Gamma_{i, j} \times\right] 0, T[, \\ \lambda_{i}\left(u_{i}\right) \nabla\left(\pi_{i}\left(u_{i}\right)\right) \cdot \mathbf{n}_{i}+\lambda_{j}\left(u_{j}\right) \nabla\left(\pi_{j}\left(u_{j}\right)\right) \cdot \mathbf{n}_{j}=0 & \text { on } \left.\Gamma_{i, j} \times\right] 0, T[, \\ \lambda_{i}\left(u_{i}\right) \nabla\left(\pi_{i}\left(u_{i}\right)\right) \cdot \mathbf{n}_{i}=0 & \text { on } \left.\partial \Omega_{i} \cap \partial \Omega \times\right] 0, T[, \\ u_{i}(\cdot, 0)=u_{0}(x) & \text { in } \Omega_{i} .\end{cases}
$$

All the results presented in this paper still hold if one does not neglect the effect of gravity and if one assumes that the global pressure is known, that is, for problems of the type

$$
\begin{cases}\phi_{i} \partial_{t} u_{i}+\nabla \cdot\left(\mathbf{q} f_{i}\left(u_{i}\right)+\lambda_{i}\left(u_{i}\right)\left(\rho_{o}-\rho_{w}\right) \mathbf{g}-\lambda_{i}\left(u_{i}\right) \nabla \pi_{i}\left(u_{i}\right)\right)=0 & \text { in } \left.\Omega_{i} \times\right] 0, T[, \\ \tilde{\pi}_{i}\left(u_{i}\right) \cap \tilde{\pi}_{j}\left(u_{j}\right) \neq \emptyset & \text { on } \left.\Gamma_{i, j} \times\right] 0, T[, \\ \sum_{k=i, j}\left(\mathbf{q} f_{k}\left(u_{k}\right)+\lambda_{k}\left(u_{k}\right)\left(\rho_{o}-\rho_{w}\right) \mathbf{g}-\lambda_{k}\left(u_{k}\right) \nabla \pi_{k}\left(u_{k}\right)\right) \cdot \mathbf{n}_{k}=0 & \text { on } \left.\Gamma_{i, j} \times\right] 0, T[, \\ \left(\mathbf{q} f_{i}\left(u_{i}\right)+\lambda_{i}\left(u_{i}\right)\left(\rho_{o}-\rho_{w}\right) \mathbf{g}-\lambda_{i}\left(u_{i}\right) \nabla \pi_{i}\left(u_{i}\right)\right) \cdot \mathbf{n}_{i}=0 & \text { on } \left.\partial \Omega_{i} \cap \partial \Omega \times\right] 0, T[, \\ u_{i}(\cdot, 0)=u_{0}(x) & \text { in } \Omega_{i},\end{cases}
$$


where $f_{i}$ is supposed to be a $C^{1}([0,1], \mathbb{R})$ increasing function, $\lambda_{i}$ is also supposed to belong to $C^{1}\left([0,1], \mathbb{R}_{+}\right)$and $\mathbf{q}$ satisfies

- $\forall i, \mathbf{q} \in\left(C^{1}\left(\bar{\Omega}_{i} \times[0, T]\right)\right)^{d}$,

- $\nabla \cdot \mathbf{q}=0$ in $\left.\Omega_{i} \times\right] 0, T[$,

- $\mathbf{q}_{\mid \Omega_{i}} \cdot \mathbf{n}_{i}+\mathbf{q}_{\mid \Omega_{j}} \cdot \mathbf{n}_{j}=0$ on $\left.\Gamma_{i, j} \times\right] 0, T[$,

- $\mathbf{q} \cdot \mathbf{n}=0$.

In order to ensure the uniqueness result stated in Theorem 5.1, we assume the technical condition (see [2] or [27])

$$
\forall i, \quad f_{i} \circ \varphi_{i}^{-1}, \lambda_{i} \circ \varphi_{i}^{-1} \in C^{0,1 / 2}\left(\left[0, \varphi_{i}(1)\right], \mathbb{R}\right) .
$$

REMARK 1.1 In the modelling of two-phase flows, irreducible saturations are often taken into account. One can suppose that there exist $s_{i}$ and $S_{i}\left(0<s_{i}<S_{i}<1\right)$ such that $\lambda_{i}(s)=0$ if $s \notin] s_{i}, S_{i}[$. In such a case, the problem $(\mathcal{P})$ becomes strongly degenerate, but a convenient scaling eliminates this difficulty (at least if $s_{i} \leqslant u_{0} \leqslant S_{i}$ a.e. in $\Omega_{i}$ ). Moreover, the dependence of the capillary pressure on the saturation can be weak, at least for saturations not too close to 0 or 1 . Thus the effects of capillarity are often neglected for the study of flows in homogeneous porous media, leading to the Buckley-Leverett equation (see e.g. [19]). Looking for degeneracy of $u \mapsto \pi_{i}(u)$ is a more complex problem, particularly if convection is not neglected as above. Suppose for example that $\pi_{i}(u)=\varepsilon u+P_{i}$, where $P_{i}$ are constants, and let $\varepsilon$ tend 0 . Nonclassical shocks can appear at the level of the interfaces $\Gamma_{i, j}$ (see [10]). Thus the notion of entropy solution used by Adimurthi, J. Jaffré, and G. D. Veerappa Gowda [1] is not sufficient to deal with this problem. This difficulty has to be overcome when considering degenerate parabolic problems. But it seems clear that the notion of entropy solution developed by K. H. Karlsen, N. H. Risebro and J. D. Towers [20, 21, 22] is not suitable for our problem.

\section{The notion of weak solution}

In this section, we introduce the notion of weak solution to the problem $(\mathcal{P})$, which is more general than the notion of weak solution given in [14, 15]. Indeed, we are able to define such a solution even in the case of an arbitrary finite number of different homogeneous porous media. Furthermore, the notion of weak solution introduced in this paper is still available in cases where the one defined in [15] does not make sense any more. We finally show that the two notions of solution are equivalent in the case where the notion of [15] is well defined. Proving the existence of a weak solution to the problem $(\mathcal{P}$ ) in a wider case is the aim of Section 3 .

We denote by $\varphi_{i}$ the $C^{1}\left([0,1], \mathbb{R}_{+}\right)$function which naturally appears in the problem $\mathcal{P}$ and which is defined by

$$
\varphi_{i}(s)=\int_{0}^{s} \lambda_{i}(a) \pi_{i}^{\prime}(a) \mathrm{d} a \quad \forall s \in[0,1] .
$$

REMARK 2.1 The assumptions on the data ensure that $\varphi_{i}^{\prime}>0$ on $] 0,1[$, and so we can define an increasing continuous function $\varphi_{i}^{-1}:\left[0, \varphi_{i}(1)\right] \rightarrow[0,1]$.

We are now able to define the notion of weak solution to the problem $(\mathcal{P})$.

Definition 2.1 Under Assumptions 1 and 2 a function $u$ is said to be a weak solution to the problem $(\mathcal{P})$ if 
1. $u \in L^{\infty}(\Omega \times] 0, T[), 0 \leqslant u \leqslant 1$ a.e. in $\left.\Omega \times\right] 0, T[$,

2. for all $i \in \llbracket 1, N \rrbracket, \varphi_{i}\left(u_{i}\right) \in L^{2}\left(0, T ; H^{1}\left(\Omega_{i}\right)\right)$, where $u_{i}$ denotes the restriction of $u$ to $\Omega_{i} \times$ ]0, $T[$,

3. $\tilde{\pi}_{i}\left(u_{i}\right) \cap \tilde{\pi}_{j}\left(u_{j}\right) \neq \emptyset$ a.e. on $\left.\Gamma_{i, j} \times\right] 0, T[$,

4. for all $\psi \in \mathcal{D}(\bar{\Omega} \times[0, T[)$,

$$
\begin{aligned}
\sum_{i=1}^{N} \int_{\Omega_{i}} \int_{0}^{T} \phi_{i} u_{i}(x, t) \partial_{t} \psi(x, t) \mathrm{d} x \mathrm{~d} t & +\sum_{i=1}^{N} \int_{\Omega_{i}} \phi_{i} u_{0}(x) \psi(x, 0) \mathrm{d} x \\
& -\sum_{i=1}^{N} \int_{\Omega_{i}} \int_{0}^{T} \nabla \varphi_{i}\left(u_{i}(x, t)\right) \cdot \nabla \psi(x, t) \mathrm{d} x \mathrm{~d} t=0 .
\end{aligned}
$$

The third point of the definition, which ensures the continuity in the graph sense of the capillary pressures on the interfaces between several porous media, is well defined. Indeed, since $\varphi_{i}\left(u_{i}\right)$ belongs to $L^{2}\left(0, T ; H^{1}\left(\Omega_{i}\right)\right)$, it admits a trace still denoted $\varphi_{i}\left(u_{i}\right)$ on $\left.\Gamma_{i, j} \times\right] 0, T[$. Thanks to Remark 2.1. we can define the trace of $u_{i}$ on $\left.\Gamma_{i, j} \times\right] 0, T[$.

REMARK 2.2 One can equivalently replace the third point of Definition 2.1 by the condition 3bis. $\breve{\pi}_{i}\left(u_{i}\right) \cap \breve{\pi}_{j}\left(u_{j}\right) \neq \emptyset$ a.e. on $\left.\Gamma_{i, j} \times\right] 0, T[$,

where $\breve{\pi}_{i}$ is the monotonic graph given by

$$
\breve{\pi}_{i}(s)= \begin{cases}\pi_{i}(s) & \text { if } s \in] 0,1[, \\ {\left[\min _{j}\left(\alpha_{j}\right), \alpha_{i}\right]} & \text { if } s=0, \\ {\left[\beta_{i}, \max _{j}\left(\beta_{j}\right)\right]} & \text { if } s=1 .\end{cases}
$$

We will now quickly show the equivalence between the notion of weak solution to the problem $(\mathcal{P})$ and the notion of weak solution given in [15], in the case where the latter is well defined, i.e. $N=2$ and $\max \left(\alpha_{1}, \alpha_{2}\right)=\alpha<\beta=\min \left(\beta_{1}, \beta_{2}\right)$. We denote as in [15] the truncated capillary pressures by $\hat{\pi}_{1}=\max \left(\alpha, \pi_{1}\right), \hat{\pi}_{2}=\min \left(\beta, \pi_{2}\right)$, and we introduce the problem $[\widetilde{\mathcal{P}}]$, which is treated in [15]:

$$
\begin{cases}\phi_{i} \partial_{t} u_{i}-\nabla \cdot\left(\lambda_{i}\left(u_{i}\right) \nabla \pi_{i}\left(u_{i}\right)\right)=0 & \text { in } \left.\Omega_{i} \times\right] 0, T[ \\ \hat{\pi}_{1}\left(u_{1}\right)=\hat{\pi}_{2}\left(u_{2}\right) & \text { on } \left.\Gamma_{i, j} \times\right] 0, T[ \\ \lambda_{1}\left(u_{1}\right) \nabla\left(\pi_{1}\left(u_{1}\right)\right) \cdot \mathbf{n}_{1}+\lambda_{2}\left(u_{2}\right) \nabla\left(\pi_{2}\left(u_{2}\right)\right) \cdot \mathbf{n}_{2}=0 & \text { on } \left.\Gamma_{i, j} \times\right] 0, T[ \\ \lambda_{i}\left(u_{i}\right) \nabla\left(\pi_{i}\left(u_{i}\right)\right) \cdot \mathbf{n}_{i}=0 & \text { on } \left.\partial \Omega_{i} \cap \partial \Omega \times\right] 0, T[ \\ u_{i}(\cdot, 0)=u_{0}(x) & \text { in } \Omega_{i} .\end{cases}
$$

Then it is easy to check that for all $\left(s_{1}, s_{2}\right) \in[0,1]^{2}$,

$$
\hat{\pi}_{1}\left(s_{1}\right)=\hat{\pi}_{2}\left(s_{2}\right) \Leftrightarrow \tilde{\pi}_{1}\left(s_{1}\right) \cap \tilde{\pi}_{2}\left(s_{2}\right) \neq \emptyset \Leftrightarrow \breve{\pi}_{1}\left(s_{1}\right) \cap \breve{\pi}_{2}\left(s_{2}\right) \neq \emptyset .
$$

In order to recall the definition of weak solution, we have to introduce the function

$$
\Psi:[\alpha, \beta] \rightarrow \mathbb{R}, \quad p \mapsto \int_{\alpha}^{p} \min _{j=1,2}\left(\lambda_{j} \circ \pi_{j}^{-1}(a)\right) \mathrm{d} a
$$

$\Psi$ is increasing, and for $i=1,2, \Psi \circ \hat{\pi}_{i} \circ \varphi_{i}^{-1}$ is a Lipschitz continuous function. 


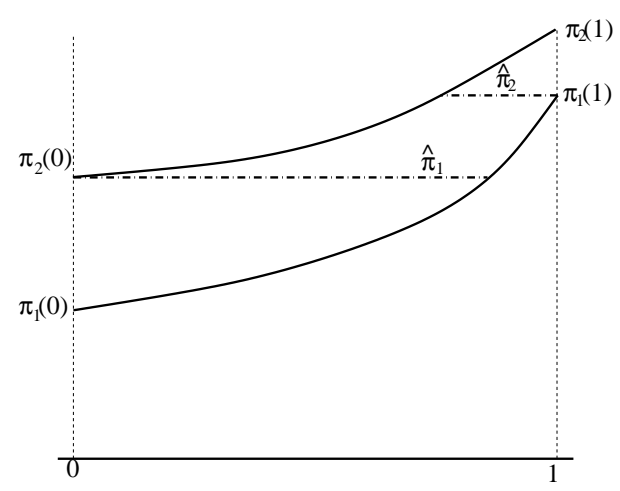

FIG. 3. Truncated capillary pressures.

DEFINITION 2.2 A function $u$ is said to be a weak solution to the problem $\widetilde{\mathcal{P}}]$ if

1. $u \in L^{\infty}(\Omega \times] 0, T[), 0 \leqslant u \leqslant 1$ a.e. in $\left.\Omega \times\right] 0, T[$,

2. $\varphi_{i}\left(u_{i}\right) \in L^{2}\left(0, T ; H^{1}\left(\Omega_{i}\right)\right)$ for $i \in\{1,2\}$,

3. $w: \Omega \times] 0, T\left[\rightarrow \mathbb{R}\right.$, defined for $\left.(x, t) \in \Omega_{i} \times\right] 0, T\left[\right.$ by $w(x, t)=\Psi \circ \hat{\pi}_{i}\left(u_{i}\right)(x, t)$, belongs to $L^{2}\left(0, T ; H^{1}(\Omega)\right)$,

4. for all $\psi \in \mathcal{D}(\bar{\Omega} \times[0, T[)$,

$$
\begin{aligned}
\sum_{i=1}^{N} \int_{\Omega_{i}} \int_{0}^{T} \phi_{i} u_{i}(x, t) \partial_{t} \psi(x, t) \mathrm{d} x \mathrm{~d} t & +\sum_{i=1}^{N} \int_{\Omega_{i}} \phi_{i} u_{0}(x) \psi(x, 0) d x \\
& -\sum_{i=1}^{N} \int_{\Omega_{i}} \int_{0}^{T} \nabla \varphi_{i}\left(u_{i}(x, t)\right) \cdot \nabla \psi(x, t) \mathrm{d} x \mathrm{~d} t=0 .
\end{aligned}
$$

REMARK 2.3 The notion of weak solution to the problem $\sqrt{\mathcal{P}}$ can be adapted to the case where there are $N>2$ homogeneous domains, but we then need compatibility conditions on $\left(\alpha_{i}\right)_{1 \leqslant i \leqslant N}$ and $\left(\beta_{i}\right)_{1 \leqslant i \leqslant N}$.

Proof of the equivalence of weak solutions. If $u$ is a weak solution to the problem $\widetilde{\mathcal{P}}$ in the sense of Definition 2.2 then for a.e. $t \in(0, T), w(\cdot, t) \in H^{1}(\Omega)$, and in particular $w(\cdot, t)$ admits a trace on $\Gamma_{i, j}$, whose value is at the same time $\Psi\left(\hat{\pi}_{i}\left(u_{i}(\cdot, t)\right)\right)$ and $\Psi\left(\hat{\pi}_{j}\left(u_{j}(\cdot, t)\right)\right)$. Since $\Psi$ is increasing, $\hat{\pi}_{i}\left(u_{i}(x, t)\right)=\hat{\pi}_{j}\left(u_{j}(x, t)\right)$ for a.e. $\left.(x, t) \in \Gamma_{i, j} \times\right] 0, T$ [. Using [14], we conclude that any weak solution to the problem $\widetilde{\mathcal{P}}$ is a weak solution to the problem $\sqrt{\mathcal{P}}$ in the sense of Definition 2.1

Conversely, if $u$ is a weak solution to the problem $\sqrt{\mathcal{P}}$ in the sense of Definition 2.1 then thanks to 114 , for almost every $\left.(x, t) \in \Gamma_{i, j} \times\right] 0, T[$,

$$
\hat{\pi}_{i}\left(u_{i}(x, t)\right)=\hat{\pi}_{j}\left(u_{j}(x, t)\right) \Leftrightarrow \Psi \circ \hat{\pi}_{i} \circ \varphi_{i}^{-1}\left(\varphi_{i}\left(u_{i}(x, t)\right)\right)=\Psi \circ \hat{\pi}_{j} \circ \varphi_{j}^{-1}\left(\varphi_{j}\left(u_{j}(x, t)\right)\right) .
$$

Since $\Psi \circ \hat{\pi}_{i} \circ \varphi_{i}^{-1}$ is a Lipschitz continuous function, the second point in Definition 2.1 ensures that $\Psi \circ \hat{\pi}_{i}\left(u_{i}\right)$ belongs to $L^{2}\left(0, T ; H^{1}\left(\Omega_{i}\right)\right)$ for $i=1,2$, and 15 ensures the continuity of the traces on $\left.\Gamma_{i, j} \times\right] 0, T$, so the third condition of Definition 2.2 is fulfilled and $u$ is a weak solution to the problem $\sqrt{\mathcal{P}}$. 
REMARK 2.4 We can define a function $\tilde{\pi}_{i}^{-1}, i \in \llbracket 1, N \rrbracket$, which satisfies $\tilde{\pi}_{i}^{-1} \circ \tilde{\pi}_{i}(s)=s$ for any $s \in[0,1]$. Using the function defined on $\mathbb{R}$ by $\tilde{\Psi}(p)=\int_{-\infty}^{p} \min _{j=1,2}\left(\lambda_{j} \circ \tilde{\pi}_{j}^{-1}(a)\right) \mathrm{d} a$, it is easy to check that we can equivalently substitute the function $\tilde{\Psi} \circ \pi_{i}\left(u_{i}\right)$ for $\Psi \circ \hat{\pi}_{i}\left(u_{i}\right)$ in the third point of Definition 2.2. This function is still defined if $\alpha \geqslant \beta$, but it becomes identically 0 , so the notion of weak solution to the problem $[\widetilde{\mathcal{P}}]$ is weaker than the notion of weak solution to the problem $(\mathcal{P})$. Indeed, $\left.u(x, t)=u_{0}(x)=a \in\right] 0,1[$ for any $(x, t) \in \Omega \times] 0, T[$ is a weak solution to the problem $\widetilde{\mathcal{P}}$, but it does not satisfy the third condition in Definition 2.1

\section{Existence of a weak solution}

The aim of this section is to prove the following theorem, which states the existence of a weak solution to the problem $(\mathcal{P})$. This result has already been proven in Section 2 in the case $N=2$ and $\alpha>\beta$, for which the notion of weak solution in the sense of Definition 2.1 is equivalent to the notion of weak solution in the sense of Definition 2.2

THEOREM 3.1 (Existence of a weak solution) Under Assumptions 1 and 2 there exists a weak solution to the problem $(\mathcal{P})$ in the sense of Definition 2.1.

Proof. We build a sequence of solutions to approximate problems (16), which converges, up to a subsequence, toward a weak solution to the problem $(\mathcal{P})$. The approximate problems do not involve capillary barriers, so existence and uniqueness of such approximate solutions is given in [9]. We leave the proof of the following technical lemma to the reader.

LEMMA 3.2 There exist sequences $\left(\lambda_{i, n}\right)_{n},\left(\pi_{i, n}\right)_{n}$ belonging to $\left(C^{\infty}([0,1], \mathbb{R})\right)^{\mathbb{N}}$ such that, for $i \in \llbracket 1, N \rrbracket$, and for $n$ large enough:

- $\lambda_{i, n \mid[0,1 / n] \cup[1-1 / n, 1]}=1 / n^{2}, \lambda_{i, n}(s)>1 / 2 n^{2}$ for all $s \in[0,1]$, and $\lambda_{i, n} \rightarrow \lambda_{i}$ uniformly on $[0,1]$

- $\pi_{i, n}(0)=\pi_{j, n}(0) \rightarrow-\infty, \pi_{i, n}(1)=\pi_{j, n}(1) \rightarrow+\infty, K n^{3 / 2}>\pi_{i, n}^{\prime} \geqslant 1 / n, \pi_{i, n} \rightarrow \pi_{i}$ in $L^{1}(0,1), \pi_{i, n} \rightarrow \pi_{i}$ and $\pi_{i, n}^{\prime} \rightarrow \pi_{i}^{\prime}$ uniformly on any compact subset of ]0, 1[,

- the function $\varphi_{i, n}: s \mapsto \int_{0}^{s} \lambda_{i, n}(a) \pi_{i, n}^{\prime}(a) \mathrm{d} a$ satisfies $\varphi_{i, n}([0,1])=\varphi_{i}([0,1])$ and $\varphi_{i, n} \rightarrow \varphi_{i}$ in $W^{1, \infty}(0,1)$.

We also define the increasing functions:

$$
\Psi_{n}:\left[a_{n}, b_{n}\right] \rightarrow \mathbb{R}, \quad p \mapsto \int_{a_{n}}^{p} \min _{j \in \mathbb{I} 1, N \rrbracket}\left(\lambda_{j, n} \circ \pi_{j, n}^{-1}(a)\right) \mathrm{d} a
$$

The conditions on the functions on the intervals $[0,1 / n] \cup[1-1 / n, 1]$ ensure that for any fixed large $n$, the functions $\left(\varphi_{i, n} \circ \pi_{i, n}^{-1} \circ \Psi_{n}^{-1}\right)^{\prime}$ are Lipschitz continuous. Then thanks to [9], for all $n$, the approximate problem

$$
\begin{cases}\phi_{i} \partial_{t} u_{i, n}-\nabla \cdot\left(\lambda_{i, n}\left(u_{i, n}\right) \nabla \pi_{i, n}\left(u_{i, n}\right)\right)=0 & \text { in } \left.\Omega_{i} \times\right] 0, T[, \\ \pi_{i, n}\left(u_{i, n}\right)=\pi_{j, n}\left(u_{j, n}\right) & \text { on } \left.\Gamma_{i, j} \times\right] 0, T[, \\ \lambda_{i, n}\left(u_{i, n}\right) \nabla\left(\pi_{i, n}\left(u_{i, n}\right)\right) \cdot \mathbf{n}_{i}+\lambda_{j, n}\left(u_{j, n}\right) \nabla\left(\pi_{j, n}\left(u_{j, n}\right)\right) \cdot \mathbf{n}_{j}=0 & \text { on } \left.\Gamma_{i, j} \times\right] 0, T[, \\ \lambda_{i, n}\left(u_{i, n}\right) \nabla\left(\pi_{i, n}\left(u_{i, n}\right)\right) \cdot \mathbf{n}_{i}=0 & \text { on } \left.\partial \Omega_{i} \cap \partial \Omega \times\right] 0, T[, \\ u_{i, n}(x, 0)=u_{0}(x) & \text { in } \Omega .\end{cases}
$$

admits a unique weak solution in the sense of Definition 3.1 below, and this solution belongs to $C\left([0, T], L^{p}(\Omega)\right)$ for $1 \leqslant p<\infty$. 
DEFINITION 3.1 A function $u_{n}$ is said to be a weak solution to the problem (16) if:

1. $u_{n} \in L^{\infty}(\Omega \times] 0, T[), 0 \leqslant u_{n} \leqslant 1$ a.e. in $\left.\Omega \times\right] 0, T[$,

2. $\varphi_{i, n}\left(u_{i, n}\right) \in L^{2}\left(0, T ; H^{1}\left(\Omega_{i}\right)\right)$ for $i \in\{1,2\}$,

3. $\left.w_{n}: \Omega \times\right] 0, T\left[\rightarrow \mathbb{R}\right.$, defined on $\left.\Omega_{i} \times\right] 0, T\left[\right.$ by $w_{n}=\Psi_{n} \circ \pi_{i, n}\left(u_{i, n}\right)$, belongs to $L^{2}\left(0, T ; H^{1}(\Omega)\right)$,

4. for all $\psi \in \mathcal{D}(\Omega \times[0, T[)$,

$$
\begin{aligned}
\sum_{i=1}^{N} \int_{\Omega_{i}} \int_{0}^{T} \phi_{i} u_{i, n}(x, t) \partial_{t} \psi(x, t) \mathrm{d} x \mathrm{~d} t & +\sum_{i=1}^{N} \int_{\Omega_{i}} \phi_{i} u_{0}(x) \psi(x, 0) \mathrm{d} x \\
& -\sum_{i=1}^{N} \int_{\Omega_{i}} \int_{0}^{T} \nabla \varphi_{i, n}\left(u_{i, n}(x, t)\right) \cdot \nabla \psi(x, t) \mathrm{d} x \mathrm{~d} t=0 .
\end{aligned}
$$

The proof of existence of a weak solution, given in [9], shows that there exists $C_{1}>0$ not depending on $n$ such that, for all $i \in \llbracket 1, N \rrbracket$ and all $n$,

$$
\left\|\varphi_{i, n}\left(u_{i, n}\right)\right\|_{L^{2}\left(0, T ; H^{1}\left(\Omega_{i}\right)\right)}^{2} \leqslant C_{1}\left\|\pi_{i, n}\right\|_{L^{1}(0,1)} ;
$$

thus $\left(\varphi_{i, n}\left(u_{i, n}\right)\right)_{n}$ is a bounded sequence in $L^{2}\left(0, T ; H^{1}\left(\Omega_{i}\right)\right)$ by Lemma 3.2. A study of the proof of the time translate estimate used in [9, 15], and detailed in [17, Lemma 4.6], leads to the existence of $C_{2}$ not depending on $n$ such that

$$
\left\|\varphi_{i, n}\left(u_{i, n}(\cdot, \cdot+\tau)\right)-\varphi_{i, n}\left(u_{i, n}(\cdot, \cdot)\right)\right\|_{L^{2}\left(\Omega_{i} \times\right] 0, T-\tau[)}^{2} \leqslant \tau C_{2}\left\|\pi_{i, n}\right\|_{L^{1}(0,1)}\left\|\varphi_{i, n}^{\prime}\right\|_{L^{\infty}(0,1)} .
$$

Lemma 3.2 and estimates (18), (19) allow us to apply Kolmogorov's compactness criterion (see e.g. [8]) to deduce the relative compactness of the sequence $\left(\varphi_{i, n}\left(u_{i, n}\right)\right)_{n}$ in $L^{2}\left(\Omega_{i} \times\right] 0, T$ [). There exists $f_{i} \in L^{2}\left(0, T ; H^{1}\left(\Omega_{i}\right)\right)$ such that

$$
\begin{array}{ll}
\varphi_{i, n}\left(u_{i, n}\right) \rightarrow f_{i} & \text { in } L^{2}\left(\Omega_{i} \times\right] 0, T[), \\
\varphi_{i, n}\left(u_{i, n}\right) \rightarrow f_{i} & \text { weakly in } L^{2}\left(0, T ; H^{1}\left(\Omega_{i}\right)\right) .
\end{array}
$$

Let us now recall a very useful lemma, classically called Minty's trick, and introduced in this framework by Leray and Lions in the famous paper [25].

LEMMA 3.3 (Minty's trick) Let $\left(\phi_{n}\right)_{n}: \mathbb{R} \rightarrow \mathbb{R}$ be a sequence of nondecreasing functions and let $\phi: \mathbb{R} \rightarrow \mathbb{R}$ be a nondecreasing continuous function such that:

- $\phi_{n} \rightarrow \phi$ pointwise,

- there exists $g \in L_{\text {loc }}^{1}(\mathbb{R})$ such that $\left|\phi_{n}\right| \leqslant g$.

Let $\mathcal{O}$ be an open subset of $\mathbb{R}^{k}, k \geqslant 1$. Let $\left(u_{n}\right)_{n} \in\left(L^{\infty}(\mathcal{O})\right)^{\mathbb{N}}$, let $u \in L^{\infty}(\mathcal{O})$ and let $f \in L^{1}(\mathcal{O})$ be such that:

- $u_{n} \rightarrow u$ in the $L^{\infty}(\mathcal{O})$-weak* sense,

- $\phi_{n}\left(u_{n}\right) \rightarrow f$ in $L^{1}(\mathcal{O})$.

Then $f=\phi(u)$. 
Since $0 \leqslant u_{i, n} \leqslant 1,\left(u_{i, n}\right)_{n}$ converges up to a subsequence to $u_{i}$ in the $L^{\infty}\left(\Omega_{i} \times\right] 0, T[)$-weak* sense. $\left(\varphi_{i, n}\right)_{n}$ converges uniformly toward $\varphi_{i}$ on $[0,1]$, and we can easily check, using Minty's trick, that $f_{i}=\varphi_{i}\left(u_{i}\right) \in L^{2}\left(0, T ; H^{1}\left(\Omega_{i}\right)\right)$. Thus we can pass to the limit in the formulation 17 , to obtain the required weak formulation:

$$
\begin{aligned}
\sum_{i=1}^{N} \int_{\Omega_{i}} \int_{0}^{T} \phi_{i} u_{i}(x, t) \partial_{t} \psi(x, t) \mathrm{d} x \mathrm{~d} t & +\sum_{i=1}^{N} \int_{\Omega_{i}} \phi_{i} u_{0}(x) \psi(x, 0) \mathrm{d} x \\
& -\sum_{i=1}^{N} \int_{\Omega_{i}} \int_{0}^{T} \nabla \varphi_{i}\left(u_{i}(x, t)\right) \cdot \nabla \psi(x, t) \mathrm{d} x \mathrm{~d} t=0 .
\end{aligned}
$$

To complete the proof of Theorem 3.1 we need the convergence of the traces of the approximate solutions $\left(u_{i, n}\right)_{n}$ on $\left.\Gamma_{i, j} \times\right] 0, T\left[\right.$ toward the trace of $u_{i}$, and to verify that $\tilde{\pi}_{i}\left(u_{i}\right) \cap \tilde{\pi}_{j}\left(u_{j}\right) \neq \varnothing$ a.e. on $\left.\Gamma_{i, j} \times\right] 0, T[$.

Since $\Omega_{i}$ has a Lipschitz boundary, there exists an operator $P$, continuous from $H^{1}\left(\Omega_{i}\right)$ into $H^{1}\left(\mathbb{R}^{d}\right)$, and also from $L^{2}\left(\Omega_{i}\right)$ into $L^{2}\left(\mathbb{R}^{d}\right)$, such that $P v_{\mid \Omega_{i}}=v$ for all $v \in L^{2}\left(\Omega_{i}\right)$. Then $P$ is continuous from $H^{s}\left(\Omega_{i}\right)$ into $H^{s}\left(\mathbb{R}^{d}\right)$ for all $s \in[0,1]$. One has, for all $v \in H^{s}\left(\Omega_{i}\right)$,

$$
\|v\|_{H^{s}\left(\Omega_{i}\right)} \leqslant\|P v\|_{H^{s}\left(\mathbb{R}^{d}\right)} \leqslant\|P v\|_{H^{1}\left(\mathbb{R}^{d}\right)}^{s}\|P v\|_{L^{2}\left(\mathbb{R}^{d}\right)}^{1-s} \leqslant C\|v\|_{H^{1}\left(\Omega_{i}\right)}^{s}\|v\|_{L^{2}\left(\Omega_{i}\right)}^{1-s} .
$$

One deduces from this inequality and from (19) that for all $s \in] 0,1[$ and $\tau \in] 0, T[$, there exists $C_{3}$ not depending on $n, \tau$ such that

$$
\left\|\varphi_{i, n}\left(u_{i, n}(\cdot, \cdot+\tau)\right)-\varphi_{i, n}\left(u_{i, n}(\cdot, \cdot)\right)\right\|_{L^{2}\left(0, T-\tau ; H^{s}\left(\Omega_{i}\right)\right)}^{2} \leqslant \tau^{1-s} C_{3}
$$

For $s_{1}>s_{2}, H^{s_{1}}$ is compactly imbedded in $H^{s_{2}}$, and so estimate 201 implies that the sequence $\left(\varphi_{i, n}\left(u_{i, n}\right)\right)_{n}$ is relatively compact in $L^{2}\left(0, T ; H^{s}\left(\Omega_{i}\right)\right)$ for all $\left.s \in\right] 0,1[$. In particular, one can extract a subsequence converging toward $\varphi_{i}\left(u_{i}\right)$ in $L^{2}\left(0, T ; H^{s}\left(\Omega_{i}\right)\right)$. We can claim, using Minty's trick once again, that the traces of $\left(\varphi_{i, n}\left(u_{i, n}\right)\right)_{n}$ on $\Gamma_{i, j}$ also converge toward the trace of $\varphi_{i}\left(u_{i}\right)$, still denoted $\varphi_{i}\left(u_{i}\right)$, in $L^{2}\left(0, T ; H^{s-1 / 2}\left(\Gamma_{i, j}\right)\right)$, and in particular for almost every $\left.(x, t) \in \Gamma_{i, j} \times\right] 0, T[$. Since $\varphi_{i}$ is increasing, $\left(u_{i, n}(x, t)\right)_{n}$ converges almost everywhere on $\left.\Gamma_{i, j} \times\right] 0, T\left[\right.$ toward $u_{i}(x, t)$.

Let us now check that $\tilde{\pi}_{i}\left(u_{i}\right) \cap \tilde{\pi}_{j}\left(u_{j}\right) \neq \varnothing$ a.e. on $\left.\Gamma_{i, j} \times\right] 0, T[$. For almost every $(x, t) \in$ $\left.\Gamma_{i, j} \times\right] 0, T\left[\right.$ the sequence $\left(\pi_{i, n}\left(u_{i, n}(x, t)\right)\right)_{n}$ converges (up to a new subsequence) to $\gamma_{i}(x, t) \in \overline{\mathbb{R}}$. Since $\pi_{i, n}\left(u_{i, n}(x, t)\right)=\pi_{j, n}\left(u_{j, n}(x, t)\right)$ for all $n$, one has

$$
\left.\gamma_{i}(x, t)=\gamma_{j}(x, t) \quad \text { a.e. on } \Gamma_{i, j} \times\right] 0, T[\text {. }
$$

If $\left.u_{i}(x, t) \in\right] 0,1\left[\right.$, then $\gamma_{i}(x, t)=\pi_{i}\left(u_{i}(x, t)\right)$. If $u_{i}(x, t)=0$, then $\gamma_{i}(x, t) \leqslant \alpha_{i}$, and $\gamma_{i}(x, t) \in \tilde{\pi}_{i}(0)$. In the same way, if $u_{i}(x, t)=1$, then $\gamma_{i}(x, t) \in \tilde{\pi}_{i}(1)$.

This completes the proof of Theorem 3.1, because relation 21) ensures the connection of the traces in the sense of

$$
\left.\tilde{\pi}_{i}\left(u_{i}\right) \cap \tilde{\pi}_{j}\left(u_{j}\right) \neq \emptyset \quad \text { a.e. on } \Gamma_{i, j} \times\right] 0, T[.
$$

\section{A regularity result}

In this section and in Section 5 , we show the existence and uniqueness of a solution with bounded flux to the problem $[\mathcal{P}$ in the one-dimensional case. We give the proofs in the case where there 
are only two subdomains $\left.\Omega_{1}=\right]-1,0\left[\right.$ and $\left.\Omega_{2}=\right] 0,1[$, but a straightforward adaptation gives the same result for any finite number of $\Omega_{i}$, each of an arbitrary finite measure. We now state the main result of this section, which states the existence of a solution with bounded spatial derivatives on $\mathcal{Q}_{i}$, where $\left.\mathcal{Q}_{i}=\Omega_{i} \times\right] 0, T[$. We also set $\mathcal{Q}=]-1,1[\times] 0, T[$ and $\Gamma=\{x=0\}$.

THEOREM 4.1 (Existence of a bounded flux solution) Let $u_{0} \in L^{\infty}(-1,1), 0 \leqslant u_{0} \leqslant 1$, be such that

- $\varphi_{i}\left(u_{0}\right) \in W^{1, \infty}\left(\Omega_{i}\right)$,

- $\tilde{\pi}_{1}\left(u_{0,1}\right) \cap \tilde{\pi}_{2}\left(u_{0,2}\right) \neq \emptyset$ on $\Gamma$.

Then there exists a weak solution $u$ to the problem $(\mathcal{P})$ such that $\partial_{x} \varphi_{i}\left(u_{i}\right) \in L^{\infty}\left(\mathcal{Q}_{i}\right)$.

All the section will be devoted to the proof of this theorem. As in Section 3, we will get this existence result by taking the limit of a sequence of solutions to approximate problems 16 involving no capillary barriers, whose data have the properties stated in Lemma 3.2

Proof. We will build a sequence of approximate initial data $\left(u_{0, n}\right)$ adapted to the sequence of approximate problems.

LEMmA 4.2 Let $u_{0}$ be as in Theorem 4.1. Then there exists $\left(u_{0, n}\right)_{n}$ such that, for all $n$ :

- $0 \leqslant u_{0, n} \leqslant 1$,

- $\pi_{1, n}\left(u_{0, n, 1}\right)=\pi_{2, n}\left(u_{0, n, 2}\right)$ on $\Gamma$,

and furthermore

$$
\lim _{n \rightarrow \infty}\left\|u_{0, n}-u_{0}\right\|_{\infty}=0, \quad\left\|\partial_{x} \varphi_{i, n}\left(u_{0, n}\right)\right\|_{L^{\infty}\left(\Omega_{i}\right)} \leqslant\left\|\partial_{x} \varphi_{i}\left(u_{0}\right)\right\|_{L^{\infty}\left(\Omega_{i}\right)} .
$$

Proof. Since $\tilde{\pi}_{1}\left(u_{0,1}\right) \cap \tilde{\pi}_{2}\left(u_{0,2}\right) \neq \emptyset$, there exists $\left(a_{1, n}, a_{2, n}\right) \in[0,1]^{2}$ such that $\pi_{1, n}\left(a_{1, n}\right)=$ $\pi_{2, n}\left(a_{2, n}\right)$ and $\left|a_{1, n}-u_{0,1}\right|+\left|a_{2, n}-u_{0,2}\right| \rightarrow 0$. We set, for $x \in \Omega_{i}$,

$$
u_{0, n}(x)=\varphi_{i, n}^{-1}\left(T_{\varphi_{i}}\left[\varphi_{i}\left(u_{0}\right)+\varphi_{i, n}\left(a_{i, n}\right)-\varphi_{i}\left(u_{0, i}\right)\right]\right)
$$

where

$$
T_{\varphi_{i}}(s)= \begin{cases}s & \text { if } s \in\left[0, \varphi_{i}(1)\right]=\left[0, \varphi_{i, n}(1)\right], \\ \varphi_{i, n}(1) & \text { if } s>\varphi_{i}(1), \\ 0 & \text { if } s<0 .\end{cases}
$$

Then the sequence $\left(u_{0, n}\right)$ converges uniformly toward $u_{0}$. Moreover, for all $n, 0 \leqslant u_{0, n} \leqslant 1$ and either $\partial_{x} \varphi_{i, n}\left(u_{0, n}\right)=\partial_{x} \varphi_{i}\left(u_{0}\right)$, or $\partial_{x} \varphi_{i, n}\left(u_{0, n}\right)=0$.

By [9], the approximate problem (16) admits a unique solution $u_{n}$ which belongs to $C\left([0, T], L^{1}(\Omega)\right)$. Now, in order to get an $L^{\infty}\left(\mathcal{Q}_{i}\right)$-estimate of the sequence $\left(\partial_{x} \varphi_{i, n}\left(u_{n}\right)\right)_{n}$, we introduce a new family of approximate problems $(23)$ for which the spatial dependence of the data is smooth.

Let $\theta \in C^{\infty}(\mathbb{R})$ with $0 \leqslant \theta \leqslant 1, \theta(x)=0$ if $x<-1$, and $\theta(x)=1$ if $x>1$. For all $k \in \mathbb{N}^{\star}$, we set

$$
\begin{aligned}
\phi^{k}(x) & =(1-\theta(k x)) \phi_{1}+\theta(k x) \phi_{2}, \\
\lambda_{n, k}(s, x) & =(1-\theta(k x)) \lambda_{1, n}(s)+\theta(k x) \lambda_{2, n}(s), \\
\pi_{n, k}(s, x) & =(1-\theta(k x)) \pi_{1, n}(s)+\theta(k x) \pi_{2, n}(s) .
\end{aligned}
$$


We will now take a new approximation of the initial data. We set

$$
u_{0, n, k}(x)= \begin{cases}u_{0, n}\left(\frac{k}{k-1}\left(x+\frac{1}{k}\right)\right) & \text { if } x<-1 / k \\ u_{0, n}\left(\frac{k}{k-1}\left(x-\frac{1}{k}\right)\right) & \text { if } x>1 / k\end{cases}
$$

In the layer $[-1 / k, 1 / k], u_{0, n, k}$ is defined by the relation

$$
(1-\theta(k x)) \pi_{1, n}\left(u_{0, n, k}(x)\right)+\theta(k x) \pi_{2, n}\left(u_{0, n, k}(x)\right)=\pi_{1, n}\left(a_{1, n}\right)=\pi_{2, n}\left(a_{2, n}\right),
$$

so that the approximate capillary pressure $\pi_{n, k}\left(u_{0, n, k}, \cdot\right)$ is constant throughout the layer.

Moreover, one has either

$$
\lambda_{n, k}\left(u_{0, n, k}, x\right) \partial_{x}\left(\pi_{n, k}\left(u_{0, n, k}, x\right)\right)=\frac{k}{k-1} \partial_{x} \varphi_{i, n}\left(u_{0, n}\right) \quad \text { if }|x|>1 / k,
$$

or

$$
\partial_{x}\left(\pi_{n, k}\left(u_{0, n, k}, x\right)\right)=0 \quad \text { if }|x|<1 / k
$$

So from the definition of $u_{0, n, k}$ we directly deduce the following lemma:

LEMMA 4.3 Let $n \geqslant 1$ and $0 \leqslant u_{0, n} \leqslant 1$ with $\varphi_{i, n}\left(u_{0, n}\right) \in W^{1, \infty}\left(\Omega_{i}\right)$ and $\pi_{1, n}\left(u_{0, n, 1}\right)=$ $\pi_{2, n}\left(u_{0, n, 2}\right)$. Then there exists a sequence $\left(u_{0, n, k}\right)_{k}$ such that, for all $k \geqslant 2,0 \leqslant u_{0, n, k} \leqslant 1$ and

$$
\left\|\lambda_{n, k}\left(u_{0, n, k}, \cdot\right) \partial_{x}\left(\pi_{n, k}\left(u_{0, n, k}, \cdot\right)\right)\right\|_{\infty} \leqslant 2 \max _{i=1,2}\left\|\partial_{x} \varphi_{i, n}\left(u_{0, n}\right)\right\|_{\infty}
$$

and

$$
u_{0, n, k} \rightarrow u_{0, n} \quad \text { in } L^{1}(\Omega) \text { as } k \rightarrow \infty .
$$

For any fixed $k \geqslant 2$ and $n$ large enough, we can now introduce the smooth nondegenerate parabolic problem

$$
\left\{\begin{array}{l}
\phi^{k}(x) \partial_{t} u_{n, k}-\partial_{x}\left(\lambda_{n, k}\left(u_{n, k}, x\right) \partial_{x} \pi_{n, k}\left(u_{n, k}, x\right)\right)=0 \\
\partial_{x} u_{n, k}(-1, t)=\partial_{x} u_{n, k}(1, t)=0 \\
u_{n, k}(x, 0)=u_{0, n, k}(x)
\end{array}\right.
$$

Moreover, one can furthermore suppose, up to a new regularization, that $u_{0, n, k} \in C^{\infty}([-1,1])$. Then (23) admits a unique strong solution $u_{n, k} \in C^{\infty}([0, T] \times[-1,1])$ (see for instance [18, 24]).

Now one sets $f_{n, k}(x, t)=\lambda_{n, k}\left(u_{n, k}, x\right) \partial_{x} \pi_{n, k}\left(u_{n, k}, x\right)$, so the main equation of 23) can be rewritten

$$
\phi^{k} \partial_{t} u_{n, k}=\partial_{x} f_{n, k}
$$

A short calculation shows that $f_{n, k}(x, t)$ is the solution of the problem

$$
\left\{\begin{array}{l}
\partial_{t} f_{n, k}=a_{n, k} \partial_{x x}^{2} f_{n, k}+b_{n, k} \partial_{x} f_{n, k} \\
f_{n, k}(-1, t)=f_{n, k}(1, t)=0 \\
f_{n, k}(x, 0)=\lambda_{n, k}\left(u_{0, n, k}, \cdot\right) \partial_{x}\left(\pi_{n, k}\left(u_{0, n, k}, \cdot\right)\right),
\end{array}\right.
$$


where $a_{n, k}, b_{n, k}$ are the regular functions defined by

$$
\begin{aligned}
& a_{n, k}=\lambda_{n, k}\left(u_{n, k}, x\right) \frac{\left(\pi_{n, k}\right)^{\prime}\left(u_{n, k}, x\right)}{\phi^{k}(x)}>0, \\
& b_{n, k}=\left(\lambda_{n, k}\right)^{\prime}\left(u_{n, k}, x\right) \frac{\partial_{x}\left[\pi_{n, k}\left(u_{n, k}, x\right)\right]}{\phi^{k}(x)}+\lambda_{n, k}\left(u_{n, k}, x\right) \partial_{x}\left[\frac{\left(\pi_{n, k}\right)^{\prime}\left(u_{n, k}, x\right)}{\phi^{k}(x)}\right] .
\end{aligned}
$$

The fact that $u_{0, n, k}$ is supposed to be regular allows us to write the problem (24) in a strong sense (this is necessary, because this problem cannot be written in a conservative form). In particular, $f_{n, k}$ satisfies the maximum principle, and thus

$$
\left\|f_{n, k}\right\|_{L^{\infty}(]-1,1[\times] 0, T[)} \leqslant\left\|\lambda_{n, k}\left(u_{0, n, k}, \cdot\right) \partial_{x}\left(\pi_{n, k}\left(u_{0, n, k}, \cdot\right)\right)\right\|_{L^{\infty}(-1,1)} .
$$

Thanks to Lemmas 4.3 and 4.2 , we have a uniform bound on $\left(f_{n, k}\right)$ :

$$
\left\|f_{n, k}\right\|_{L^{\infty}(]-1,1[\times] 0, T[)} \leqslant 2 \max _{i=1,2}\left\|\partial_{x} \varphi_{i}\left(u_{0}\right)\right\|_{\infty} .
$$

Since the problem 23 is fully nondegenerate (recall that $\lambda_{i, n}>1 / 2 n^{2}$ and $\pi_{i, n}^{\prime} \geqslant$ $1 / n)$ it follows that $\partial_{x} u_{n, k}$ and $\partial_{t} u_{n, k}$ are uniformly bounded respectively in $L^{\infty}\left(\mathcal{Q}_{i}\right)$ and in $L^{2}\left(0, T ; H^{-1}\left(\Omega_{i}\right)\right)$ with respect to $k$; then the sequence $\left(u_{n, k}\right)_{k}$ converges toward $u_{n}$ in $L^{2}\left(\mathcal{Q}_{i}\right)$, and the limit $u_{n}$ satisfies, thanks to the estimate $[25]$,

$$
\left\|\partial_{x} \varphi_{i, n}\left(u_{n}\right)\right\|_{L^{\infty}\left(\mathcal{Q}_{i}\right)} \leqslant 2 \max _{i=1,2}\left\|\partial_{x} \varphi_{i}\left(u_{0}\right)\right\|_{\infty} .
$$

For all $\psi \in \mathcal{D}([-1,1] \times[0, T[)$, one has

$$
\int_{0}^{T} \int_{-1}^{1} \phi^{k} u_{n, k} \partial_{t} \psi+\int_{-1}^{1} \phi^{k} u_{0, n}^{k} \psi_{0}-\int_{0}^{T} \int_{-1}^{1} f_{n, k} \partial_{x} \psi=0 .
$$

Thanks to (25),

$$
\lim _{k \rightarrow \infty} \int_{0}^{T} \int_{-1 / k}^{1 / k} f_{n, k} \partial_{x} \psi=0
$$

One has $u_{n, k} \rightarrow u_{n}$ in the $L^{\infty}(\mathcal{Q})$-weak ${ }^{*}$ and $L^{2}(\mathcal{Q})$ senses, and $u_{0, n, k} \rightarrow u_{0, n}$ in $L^{1}(-1,1)$ thanks to Lemma 4.3 Moreover, by $25, \partial_{x} \pi_{i, n, k}\left(u_{n, k}\right) \rightarrow \partial_{x} \pi_{i, n}\left(u_{n, k}\right)$ in the $L^{\infty}(\mathcal{Q})$-weak ${ }^{*}$ sense. Thus we can let $k$ tend toward $\infty$ in (27) to get

$$
\int_{0}^{T} \sum_{i=1,2} \int_{\Omega_{i}} \phi_{i} u_{n} \partial_{t} \psi+\sum_{i=1,2} \int_{\Omega_{i}} \phi_{i} u_{0, n} \psi_{0}-\int_{0}^{T} \sum_{i=1,2} \int_{\Omega_{i}} \lambda_{i, n}\left(u_{n}\right) \partial_{x} \pi_{i, n}\left(u_{n}\right) \partial_{x} \psi=0 .
$$

Furthermore, using the fact that $\pi_{n, k}\left(u_{n, k}, x\right)$ belongs to $L^{2}\left(0, T ; H^{1}(\Omega)\right)$ and, even more, that $\partial_{x}\left(\pi_{n, k}\left(u_{n, k}, x\right)\right)$ is bounded uniformly in $k$, we can claim that $\pi_{1, n}\left(u_{1, n}\right)=\pi_{2, n}\left(u_{2, n}\right)$, and so $u_{n}$ is the unique weak solution to the approximate problem $(16)$ with initial data $u_{0, n}$.

When $n$ tends toward $\infty$, the sequence $\left(u_{n}\right)_{n}$ converges, up to a subsequence, toward a weak solution to the problem $(\mathcal{P})$, as seen in Section 3 , and the estimate 26 ensures that

$$
\partial_{x} \varphi_{i}(u) \in L^{\infty}\left(\mathcal{Q}_{i}\right)
$$

This completes the proof of Theorem 4.1 . 


\section{A uniqueness result}

In this section, we give a uniqueness result in the one-dimensional case in a framework where the existence results are stronger than the general existence result stated in Theorem 3.1. Under a regularity assumption on the initial data $u_{0}$, we have proved in Section 4 the existence of a solution having bounded flux, for which we give a uniqueness result in Theorem 5.1 and Corollary 5.2. The bound on the flux will be necessary to prove that the contraction property is also available in the neighbourhood of the interface $\{x=0\}$. Then we show in Theorem 5.4 the existence and uniqueness of the weak solution which is the limit of bounded flux solutions for any initial data $u_{0}$ with $0 \leqslant u_{0} \leqslant 1$. Indeed, the set of initial data giving a bounded flux solution is dense in $L^{\infty}(\Omega)$ for the $L^{1}(\Omega)$ topology, and Theorem 5.1 implies that the contraction property can be extended to a larger class of solutions, defined for all initial data in $L^{\infty}(\Omega)$. Unfortunately, we can only characterize them by a limit of bounded flux solutions, and we cannot exhibit a weak solution which is not the limit of bounded flux solutions.

THEOREM 5.1 ( $L^{1}$-contraction principle for bounded flux solutions) Let $u, v$ be two weak solutions to the problem $(\mathcal{P})$ for the initial data $u_{0}, v_{0}$. Then, if $\partial_{x} \varphi_{i}\left(u_{i}\right)$ and $\partial_{x} \varphi_{i}\left(v_{i}\right)$ belong to $L^{\infty}\left(\mathcal{Q}_{i}\right)$, we have the following $L^{1}$-contraction principle: for all $t \in[0, T]$,

$$
\sum_{i=1,2} \int_{\Omega_{i}} \phi_{i}(u(x, t)-v(x, t))^{ \pm} \mathrm{d} x \leqslant \sum_{i=1,2} \int_{\Omega_{i}} \phi_{i}\left(u_{0}(x)-v_{0}(x)\right)^{ \pm} \mathrm{d} x .
$$

The first part of this section is devoted to the proof of Theorem 5.1. which, with Theorem 4.1. admits the following straightforward consequence:

COROLlary 5.2 (Uniqueness of the bounded flux solution) For all $u_{0} \in L^{\infty}(-1,1)$ with $0 \leqslant$ $u_{0} \leqslant 1$ such that $\varphi_{i}\left(u_{0}\right) \in W^{1, \infty}\left(\Omega_{i}\right)$ for $i=1,2$ and $\tilde{\pi}_{1}\left(u_{0,1}\right) \cap \tilde{\pi}_{2}\left(u_{0,2}\right) \neq \emptyset$, there exists a unique weak solution to the problem $(\mathcal{P})$ in the sense of Definition 2.1 and such that $\partial_{x} \varphi_{i}(u) \in L^{\infty}\left(\mathcal{Q}_{i}\right)$; moreover, $u \in C\left([0, T], L^{p}(\Omega)\right)$ for all $1 \leqslant p<\infty$.

Proof. The proof of Theorem 5.1 is based on entropy inequalities, obtained through the method of doubling variables, first introduced by S. Kružkov [23] for first order equations, and then adapted by J. Carrillo [11] to degenerate parabolic problems. Note that in the present setting, we only need doubling with respect to the time variable, as, for instance, in F. Otto [27] for elliptic-parabolic problems (or in [7] for Stefan-type problems).

We will only give the comparison

$$
\sum_{i=1,2} \int_{\Omega_{i}} \phi_{i}(u(x, t)-v(x, t))^{+} \mathrm{d} x \leqslant \sum_{i=1,2} \int_{\Omega_{i}} \phi_{i}\left(u_{0}(x)-v_{0}(x)\right)^{+} \mathrm{d} x .
$$

The comparison with $(\cdot)^{-}$instead of $(\cdot)^{+}$can be proven exactly the same way.

Let $u$ be a bounded flux solution to the one-dimensional problem, i.e. $\partial_{x} \varphi_{i}(u) \in L^{\infty}\left(\mathcal{Q}_{i}\right)$, $i=1$, 2. The weak formulation of Definition 2.1 adapted to the one-dimensional framework of this section can be rewritten, for all $\psi \in \mathcal{D}(\bar{\Omega} \times[0, T[)$,

$$
\begin{aligned}
\int_{0}^{T} \sum_{i=1,2} \int_{\Omega_{i}} \phi_{i} u(x, t) \partial_{t} \psi(x, t) \mathrm{d} x \mathrm{~d} t & +\sum_{i=1,2} \int_{\Omega_{i}} \phi_{i} u_{0}(x) \psi(x, 0) \mathrm{d} x \\
& -\int_{0}^{T} \sum_{i=1,2} \int_{\Omega_{i}} \partial_{x} \varphi_{i}(u)(x, t) \partial_{x} \psi(x, t) \mathrm{d} x \mathrm{~d} t=0 .
\end{aligned}
$$


This formulation clearly implies, for $i=1,2$, and all $\psi \in C_{c}^{\infty}\left(\bar{\Omega}_{i} \times[0, T[)\right.$ with $\psi(0, t)=0$,

$$
\begin{aligned}
\int_{0}^{T} \int_{\Omega_{i}} \phi_{i} u(x, t) \partial_{t} \psi(x, t) \mathrm{d} x \mathrm{~d} t & +\int_{\Omega_{i}} \phi_{i} u_{0}(x) \psi(x, 0) \mathrm{d} x \\
& \quad-\int_{0}^{T} \int_{\Omega_{i}} \partial_{x} \varphi_{i}(u)(x, t) \partial_{x} \psi(x, t) \mathrm{d} x \mathrm{~d} t=0 .
\end{aligned}
$$

Classical computations (see e.g. [7, 11, 27]) on equation (31) lead to the following entropy inequalities: for all weak solutions $u, v$, initial data $u_{0}, v_{0}$, and $\xi \in \mathcal{D}^{+}\left(\bar{\Omega}_{i} \times[0, T[\times[0, T[)\right.$ such that $\xi(0, t, s)=0$,

$$
\begin{aligned}
\int_{0}^{T} \int_{0}^{T} \int_{\Omega_{i}} \phi_{i} & (u(x, t)-v(x, s))^{+}\left(\partial_{t} \xi(x, t, s)+\partial_{s} \xi(x, t, s)\right) \mathrm{d} x \mathrm{~d} t \mathrm{~d} s \\
& +\int_{0}^{T} \int_{\Omega_{i}} \phi_{i}\left(u_{0}(x)-v(x, s)\right)^{+} \xi(x, 0, s) \mathrm{d} x \mathrm{~d} s \\
& +\int_{0}^{T} \int_{\Omega_{i}} \phi_{i}\left(u(x, t)-v_{0}(x)\right)^{+} \xi(x, t, 0) \mathrm{d} x \mathrm{~d} t \\
& -\int_{0}^{T} \int_{0}^{T} \int_{\Omega_{i}} \partial_{x}\left(\varphi_{i}(u)(x, t)-\varphi_{i}(v)(x, s)\right)^{+} \partial_{x} \xi(x, t, s) \mathrm{d} x \mathrm{~d} t \mathrm{~d} s \geqslant 0 .
\end{aligned}
$$

Let us note here an important consequence of the entropy inequality (32) (and of the corresponding one for $\left.(u-v)^{-}\right)$, namely that $u$ can be proved to satisfy

$$
\underset{t \rightarrow 0}{\operatorname{ess}-\lim } \int_{\Omega_{i}}\left|u(x, t)-u_{0}(x)\right| \mathrm{d} x=0 .
$$

Indeed, this follows by taking $v$ to be a constant in (32) and using an approximation argument (see e.g. Lemma 7.41 in [26]). We deduce the time continuity at $t=0$ for any solution and in particular for both $u$ and $v$ taken above.

Now, let $\rho \in C_{c}^{\infty}\left(\mathbb{R}, \mathbb{R}^{+}\right)$with $\operatorname{supp}(\rho) \subset[-1,1]$ and $\int_{\mathbb{R}} \rho(t) \mathrm{d} t=1$. Define $\rho_{m}(t)=m \rho(m t)$. Let $\psi \in \mathcal{D}^{+}\left([-1,1] \times\left[0, T[)\right.\right.$ with $\psi(0, \cdot)=0$. For $m$ large enough, $\xi(x, t, s)=\psi(x, t) \rho_{m}(t-s)$ belongs to $\mathcal{D}^{+}([-1,1] \times[0, T[\times[0, T[)$, and we can take it as a test function in $(32)$. Then summing on $i=1,2$ leads to

$$
\begin{aligned}
& \int_{0}^{T} \int_{0}^{T} \sum_{i=1,2} \int_{\Omega_{i}} \phi_{i}(u(x, t)-v(x, s))^{+} \partial_{t} \psi(x, t) \rho_{m}(t-s) \mathrm{d} x \mathrm{~d} t \mathrm{~d} s \\
& \quad+\int_{0}^{T} \sum_{i=1,2} \int_{\Omega_{i}} \phi_{i}\left(u_{0}(x)-v(x, s)\right)^{+} \psi(x, 0) \rho_{m}(-s) \mathrm{d} x \mathrm{~d} s \\
& \quad+\int_{0}^{T} \sum_{i=1,2} \int_{\Omega_{i}} \phi_{i}\left(u(x, t)-v_{0}(x)\right)^{+} \psi(x, t) \rho_{m}(t) \mathrm{d} x \mathrm{~d} t \\
& \quad-\int_{0}^{T} \int_{0}^{T} \sum_{i=1,2} \int_{\Omega_{i}} \partial_{x}\left(\varphi_{i}(u)(x, t)-\varphi_{i}(v)(x, s)\right)^{+} \partial_{x} \psi(x, t) \rho_{m}(t-s) \mathrm{d} x \mathrm{~d} t \mathrm{~d} s \geqslant 0 .
\end{aligned}
$$


We can now let $m$ tend toward $\infty$ in (34), and using (33) for $u$ and $v$, and the theorem of continuity in the mean, we get: for all $\psi \in \mathcal{D}^{+}(\bar{\Omega} \times[0, T[)$ such that $\psi(0, t)=0$,

$$
\begin{aligned}
\int_{0}^{T} \sum_{i=1,2} \int_{\Omega_{i}} \phi_{i}(u(x, t)-v(x, t))^{+} \partial_{t} \psi(x, t) \mathrm{d} x \mathrm{~d} t \\
+\sum_{i=1,2} \int_{\Omega_{i}} \phi_{i}\left(u_{0}(x)-v_{0}(x)\right)^{+} \psi(x, 0) \mathrm{d} x \\
-\int_{0}^{T} \sum_{i=1,2} \int_{\Omega_{i}} \partial_{x}\left(\varphi_{i}(u)(x, t)-\varphi_{i}(v)(x, t)\right)^{+} \partial_{x} \psi(x, t) \mathrm{d} x \mathrm{~d} t \geqslant 0 .
\end{aligned}
$$

We now aim to extend the inequality 35 to the case where $\psi(0, t) \neq 0$, and in particular to the case $\psi(x, t)=\theta(t)$, so that the third term disappears in 35 .

To this end, let $u_{i}(t)=u_{i}(0, t)$ denote the trace of $u_{i}$ at the interface $\Gamma$ (and correspondingly, $\left.v_{i}(t)=v_{i}(0, t)\right)$. We introduce the following subsets of $] 0, T[$ :

$$
\begin{aligned}
& E_{u>v}=\left\{t \in[0, T] \mid u_{1}(t)>v_{1}(t) \text { or } u_{2}(t)>v_{2}(t)\right\}, \\
& E_{u \leqslant v}=\left\{t \in[0, T] \mid u_{1}(t) \leqslant v_{1}(t) \text { and } u_{2}(t) \leqslant v_{2}(t)\right\},
\end{aligned}
$$

so that $E_{u \leqslant v}$ is the complement of $E_{u>v}$ in [0,T].

For all $\varepsilon>0$, one defines $\psi_{\varepsilon}(x)=\max (1-|x| / \varepsilon, 0)$. For all $\theta \in \mathcal{D}^{+}([0, T[)$, we take $(x, t) \mapsto \theta(t)\left(1-\psi_{\varepsilon}(x)\right)$ instead of $\psi(x, t)$ as a test function in (35), to get

$$
\begin{aligned}
& \int_{0}^{T} \sum_{i=1,2} \int_{\Omega_{i}} \phi_{i}(u(x, t)-v(x, t))^{+} \partial_{t} \theta(t)\left(1-\psi_{\varepsilon}(x)\right) \mathrm{d} x \mathrm{~d} t \\
& \quad+\sum_{i=1,2} \int_{\Omega_{i}} \phi_{i}\left(u_{0}(x)-v_{0}(x)\right)^{+}\left(1-\psi_{\varepsilon}\right)(x) \theta(0) \mathrm{d} x \\
& \quad-\int_{0}^{T} \frac{\theta(t)}{\varepsilon}\left(\begin{array}{c}
\left(\varphi_{1}(u)(-\varepsilon, t)-\varphi_{1}(v)(-\varepsilon, t)\right)^{+}-\left(\varphi_{1}\left(u_{1}\right)(t)-\varphi_{1}\left(v_{1}\right)(t)\right)^{+} \\
+\left(\varphi_{2}(u)(\varepsilon, t)-\varphi_{2}(v)(\varepsilon, t)\right)^{+}-\left(\varphi_{2}\left(u_{2}\right)(t)-\varphi_{2}\left(v_{2}\right)(t)\right)^{+}
\end{array}\right) \mathrm{d} t \geqslant 0 .
\end{aligned}
$$

For almost every $t \in E_{u \leqslant v}$, the function $\left(\varphi_{i}(u)-\varphi_{i}(v)\right)^{+}(\cdot, t)$ admits a nil trace on $\{x=0\}$, thus the third term in the previous inequality can be reduced to the set $E_{u>v}$, yielding

$$
\begin{aligned}
\int_{0}^{T} \sum_{i=1,2} \int_{\Omega_{i}} \phi_{i}(u(x, t)-v(x, t))^{+} \partial_{t} \theta(t)\left(1-\psi_{\varepsilon}(x)\right) \mathrm{d} x \mathrm{~d} t \\
+\sum_{i=1,2} \int_{\Omega_{i}} \phi_{i}\left(u_{0}(x)-v_{0}(x)\right)^{+}\left(1-\psi_{\varepsilon}\right)(x) \theta(0) \mathrm{d} x \\
+\int_{E_{u>v}} \theta(t) \sum_{i=1,2} \int_{\Omega_{i}} \partial_{x}\left(\varphi_{i}(u)(x, t)-\varphi_{i}(v)(x, t)\right)^{+} \partial_{x} \psi_{\varepsilon}(x) \mathrm{d} x \mathrm{~d} t \geqslant 0 .
\end{aligned}
$$

We now turn to the crucial point of the uniqueness proof, which is the following lemma. 
LEMMA 5.3 For all $\theta \in \mathcal{D}^{+}([0, T[)$, if $u, v$ are both bounded flux solutions, that is, $\partial_{x} \varphi_{i}(u), \partial_{x} \varphi_{i}(v) \in L^{\infty}\left(\mathcal{Q}_{i}\right)$, then

$$
\limsup _{\varepsilon \rightarrow 0} \int_{E_{u>v}} \theta(t) \sum_{i=1,2} \int_{\Omega_{i}} \partial_{x}\left(\varphi_{i}(u)(x, t)-\varphi_{i}(v)(x, t)\right)^{+} \partial_{x} \psi_{\varepsilon}(x) \mathrm{d} x \mathrm{~d} t \leqslant 0 .
$$

Using the weak formulation $[30)$, we can claim that for any $\vartheta \in \mathcal{D}([0, T[)$,

$$
\lim _{\varepsilon \rightarrow 0} \int_{0}^{T} \vartheta(t) \sum_{i=1,2} \int_{\Omega_{i}} \partial_{x}\left(\varphi_{i}(u)-\varphi_{i}(v)\right) \partial_{x} \psi_{\varepsilon}(x) \mathrm{d} x \mathrm{~d} t=0 .
$$

Since $\partial_{x}\left(\varphi_{i}(u)-\varphi_{i}(v)\right)$ belongs to $L^{\infty}\left(\Omega_{i} \times\right] 0, T[)$ for $i=1,2$, one has

$$
\left|\int_{0}^{T} \vartheta(t) \sum_{i=1,2} \int_{\Omega_{i}} \partial_{x}\left(\varphi_{i}(u)-\varphi_{i}(v)\right) \partial_{x} \psi_{\varepsilon}(x) \mathrm{d} x \mathrm{~d} t\right| \leqslant C\|\vartheta\|_{L^{1}(0, T)} .
$$

Then a density argument shows that 37 still holds for any $\vartheta \in L^{1}(0, T)$, and in particular for $\vartheta(t)=\theta(t) \mathbb{1}_{E_{u>v}}(t)$. Thus there exists $A(\varepsilon)$ tending to 0 as $\varepsilon$ tends to 0 such that

$$
\int_{E_{u>v}} \theta(t) \sum_{i=1,2} \int_{\Omega_{i}} \partial_{x}\left(\varphi_{i}(u)(x, t)-\varphi_{i}(v)(x, t)\right) \partial_{x} \psi_{\varepsilon}(x) \mathrm{d} x \mathrm{~d} t=A(\varepsilon) .
$$

If we split up $\varphi_{i}(u)(x, t)-\varphi_{i}(v)(x, t)$ into the positive and negative parts, 38) becomes

$$
\begin{aligned}
\int_{E_{u>v}} \theta(t) & \sum_{i=1,2} \int_{\Omega_{i}} \partial_{x}\left(\varphi_{i}(u)(x, t)-\varphi_{i}(v)(x, t)\right)^{+} \partial_{x} \psi_{\varepsilon}(x) \mathrm{d} x \mathrm{~d} t \\
& =\int_{E_{u>v}} \theta(t) \sum_{i=1,2} \int_{\Omega_{i}} \partial_{x}\left(\varphi_{i}(u)(x, t)-\varphi_{i}(v)(x, t)\right)^{-} \partial_{x} \psi_{\varepsilon}(x) \mathrm{d} x \mathrm{~d} t+A(\varepsilon) .
\end{aligned}
$$

It is at this point that we actually use the monotonicity of the transmission condition, i.e. condition 3 in Definition 2.1. Indeed, the conditions $\tilde{\pi}_{1}\left(u_{1}(t)\right) \cap \tilde{\pi}_{2}\left(u_{2}(t)\right) \neq \emptyset$ and $\tilde{\pi}_{1}\left(v_{1}(t)\right) \cap \tilde{\pi}_{2}\left(v_{2}(t)\right) \neq \emptyset$ ensure that

$$
u_{1}>v_{1} \Rightarrow u_{2} \geqslant v_{2} \text { and } u_{1}<v_{1} \Rightarrow u_{2} \leqslant v_{2} .
$$

Therefore, recalling the definition of the set $E_{u>v}$ and of $\psi_{\varepsilon}$, the first term in the right member of 39 ) is nonpositive, and we conclude

$$
\limsup _{\varepsilon \rightarrow 0} \int_{E_{u>v}} \theta(t) \sum_{i=1,2} \int_{\Omega_{i}} \partial_{x}\left(\varphi_{i}(u)(x, t)-\varphi_{i}(v)(x, t)\right)^{+} \partial_{x} \psi_{\varepsilon}(x) \mathrm{d} x \mathrm{~d} t \leqslant 0 .
$$

This completes the proof of Lemma 5.3 , and allows us to let $\varepsilon \rightarrow 0$ in inequality (36). Then for all $\psi \in \mathcal{D}^{+}([0, T[)$, one gets

$$
-\int_{0}^{T} \sum_{i=1,2} \int_{\Omega_{i}} \phi_{i}(u(x, t)-v(x, t))^{+} \partial_{t} \psi(t) \mathrm{d} x \mathrm{~d} t \leqslant \sum_{i=1,2} \int_{\Omega_{i}} \phi_{i}\left(u_{0}(x)-v_{0}(x)\right)^{+} \psi(0) \mathrm{d} x .
$$


One can also prove in exactly the same way that

$$
-\int_{0}^{T} \sum_{i=1,2} \int_{\Omega_{i}} \phi_{i}(u(x, t)-v(x, t))^{-} \partial_{t} \psi(t) \mathrm{d} x \mathrm{~d} t \leqslant \sum_{i=1,2} \int_{\Omega_{i}} \phi_{i}\left(u_{0}(x)-v_{0}(x)\right)^{-} \psi(0) \mathrm{d} x .
$$

These inequalities still hold for $\psi=T-t$, and then if $u_{0}=v_{0}$, one has $u=v$ almost everywhere in $\mathcal{Q}$. Moreover, we can take $\psi(t)=\mathbb{1}_{[0, s]}(t)$ as a test function in 41 to get the $L^{1}$-contraction principle 29, stated in Theorem 5.1

In the following, we prove that for any $u_{0}$ in $L^{\infty}(-1,1)$ with $0 \leqslant u_{0} \leqslant 1$, there exists a unique weak solution of the problem $\left(\mathcal{P}\right.$ which is the limit of a sequence $\left(u_{n}\right)_{n}$ of bounded flux solutions, i.e. $\partial_{x} \varphi_{i}\left(u_{n}\right) \in L^{\infty}\left(\mathcal{Q}_{i}\right)$ for all $n \geqslant 1$.

THEOREM 5.4 (Existence and uniqueness of the SOLA) Let $u_{0} \in L^{\infty}(-1,1), 0 \leqslant u_{0} \leqslant 1$, and let $\left(u_{0, n}\right)_{n \geqslant 1}$ be a sequence of bounded flux initial data, i.e. for all $n \geqslant 1$,

- $0 \leqslant u_{0, n} \leqslant 1$,

- $\varphi_{i}\left(u_{0, n}\right) \in W^{1, \infty}\left(\Omega_{i}\right)$,

- $\tilde{\pi}_{1}\left(u_{0, n, 1}\right) \cap \tilde{\pi}_{2}\left(u_{0, n, 2}\right) \neq \emptyset$,

such that

$$
\lim _{n \rightarrow \infty}\left\|u_{0, n}-u_{0}\right\|_{L^{1}(\Omega)}=0 .
$$

Let $\left(u_{n}\right)_{n \geqslant 1}$ be the sequence of the bounded flux solutions to the problem $\mathcal{P}$ for $u_{0, n}$ as initial data. Then the sequence $\left(u_{n}\right)_{n \geqslant 1}$ converges toward $u$ in $C(] 0, T\left[, L^{p}(-1,1)\right), 1 \leqslant p<\infty$, where $u$ is a solution to the problem $(\mathcal{P})$, called a Solution Obtained as the Limit of Approximations (SOLA). Furthermore, if $u, v$ are two SOLAs, for initial data $u_{0}, v_{0}$, one has the following $L^{1}$-contraction principle: for all $t \in[0, T]$,

$$
\sum_{i=1}^{N} \int_{\Omega_{i}} \phi_{i}(u(x, t)-v(x, t))^{ \pm} \mathrm{d} x \leqslant \sum_{i=1}^{N} \int_{\Omega_{i}} \phi_{i}\left(u_{0}(x)-v_{0}(x)\right)^{ \pm} \mathrm{d} x .
$$

This in particular leads to the uniqueness of the SOLA.

Proof. Let $\left(u_{0, n}\right)$ be a regular sequence of initial data converging toward $u_{0}$ in $L^{1}(-1,1)$; one can take e.g. $u_{0, n} \in C_{c}^{\infty}(]-1,0[\cup] 0,1[)$. Then $\left(u_{0, n}\right)$ is a Cauchy sequence, and thanks to 29 , for all $t \in[0, T]$,

$$
\sum_{i=1}^{N} \int_{\Omega_{i}} \phi_{i}\left|u_{n}(x, t)-u_{m}(x, t)\right| \mathrm{d} x \leqslant \sum_{i=1}^{N} \int_{\Omega_{i}} \phi_{i}\left|u_{0, n}(x)-u_{0, m}(x)\right| \mathrm{d} x .
$$

Thus $\left(u_{n}\right)_{n}$ is a Cauchy sequence in $C\left([0, T], L^{1}(\Omega)\right)$ and converges to a function $u$ in $C\left([0, T], L^{1}(\Omega)\right)$. Since $\left(u_{n}\right)_{n}$ is bounded in $L^{\infty}(\mathcal{Q})$, one has $u_{n} \rightarrow u$ in $C\left([0, T], L^{p}(-1,1)\right)$.

We now have to check that $u$ is a weak solution to the problem $(\mathcal{P})$. It is easy to check, using the $L^{\infty}$-bound of $u_{n}$, that $\varphi_{i}\left(u_{n}\right)$ tends toward $\varphi_{i}(u)$ in $L^{p}\left(\Omega_{i} \times\right] 0, T[)$ for all $p \in[1, \infty[$. Thanks to 18$)$, the sequence $\left(\varphi_{i}\left(u_{n}\right)\right)_{n}$ is bounded in $L^{2}\left(0, T ; H^{1}\left(\Omega_{i}\right)\right)$, and thus $\varphi_{i}\left(u_{n}\right) \rightarrow \varphi_{i}(u)$ weakly in $L^{2}\left(0, T ; H^{1}\left(\Omega_{i}\right)\right)$, and $\varphi_{i}\left(u_{n}\right)$ converges in $L^{2}\left(0, T ; H^{s}\left(\Omega_{i}\right)\right)$, for all $\left.s \in\right] 0,1[$, still toward 
$\varphi_{i}(u)$. In particular, $u_{n, i}(t)$ tends toward $u_{i}(t)$. Since the set $\left\{(a, b) \in[0,1]^{2} \mid \tilde{\pi}_{1}(a) \cap \tilde{\pi}_{2}(b) \neq \emptyset\right\}$ is closed, we can claim that

$$
\tilde{\pi}_{1}\left(u_{1}(t)\right) \cap \tilde{\pi}_{2}\left(u_{2}(t)\right) \neq \varnothing \quad \text { for a.e. } t \in[0, T] .
$$

We can also pass to the limit in the weak formulation in order to conclude that $u$ is a weak solution to the problem $(\mathcal{P})$, proving the existence of a SOLA $u$.

Let now $v$ be another SOLA, obtained through a sequence $\left(v_{0, n}\right)_{n}$ of regular initial data converging toward $v_{0}$. Thanks to 29 , one has

$$
\sum_{i=1}^{N} \int_{\Omega_{i}} \phi_{i}\left|u_{n}(x, t)-v_{n}(x, t)\right| \mathrm{d} x \leqslant \sum_{i=1}^{N} \int_{\Omega_{i}} \phi_{i}\left|u_{0, n}(x)-v_{0, n}(x)\right| \mathrm{d} x,
$$

whose limit as $n$ tends toward $\infty$ gives the desired $L^{1}$-contraction principle:

$$
\sum_{i=1}^{N} \int_{\Omega_{i}} \phi_{i}|u(x, t)-v(x, t)| \mathrm{d} x \leqslant \sum_{i=1}^{N} \int_{\Omega_{i}} \phi_{i}\left|u_{0}(x)-v_{0}(x)\right| \mathrm{d} x,
$$

and so the uniqueness of the SOLA, completing the proof of Theorem 5.4

\section{REFERENCES}

1. Adimurthi, Jaffré, J., \& Veerappa Gowda, G. D. Godunov-type methods for conservation laws with a flux function discontinuous in space. SIAM J. Numer. Anal. 42 (2004), 179-208. Zbl 1081.65082 MR 2051062

2. Alt, H. W., \& Luckhaus, S. Quasilinear elliptic-parabolic differential equations. Math. Z. 183 (1983), 311-341. Zbl 0497.35049 MR 0706391

3. Antontsev, S. N., Kazhikhov, A. V., \& Monakhov, V. N. Boundary Value Problems in Mechanics of Nonhomogeneous Fluids. Stud. Math. Appl. 17, North-Holland, Amsterdam (1990). Zbl 0696.76001 MR 1035212

4. AzIZ, K., \& Settari, A. Petroleum Reservoir Simulation. Elsevier, London (1979).

5. BeAR, J. Dynamic of Fluids in Porous Media. Dover, New York (1972).

6. Bertsch, M., PASso, R. D., \& VAN DuiJn, C. Analysis of oil trapping in porous media flow. SIAM J. Math. Anal. 35 (2003), 245-267. Zbl 1049.35108 MR 2001474

7. Blanchard, D., \& Porretta, A. Stefan problems with nonlinear diffusion and convection. $J$. Differential Equations 210 (2005), 383-428. Zbl 1075.35112 MR 2119989

8. BrÉZIS, H. Analyse fonctionnelle: Théorie et applications. Masson, Paris (1983). Zbl 0511.46001 MR 0697382

9. CANCÈs, C. Nonlinear parabolic equations with spatial discontinuities. Nonlinear Differential Equations Appl. 15 (2008), 427-456. MR 2465972

10. CANCÈs, C. Asymptotic behavior of two-phase flows in heterogeneous porous media for capillarity depending only on the space I, II. Submitted, arXiv:09021877, 0902.1872.

11. CARrillo, J. Entropy solutions for nonlinear degenerate problems. Arch. Ration. Mech. Anal. 147 (1999), 269-361. Zbl 0935.35056 MR 1709116

12. Chavent, G., \& JAfFré, J. Mathematical Models and Finite Elements for Reservoir Simulation. Stud. Math. Appl. 17, North-Holland, Amsterdam (1986). Zbl 0603.76101

13. van Duijn, C. J., MolenaAr, J., \& DE Neef, M. J. The effect of capillary forces on immiscible two-phase flows in heterogeneous porous media. Transport in Porous Media 21 (1995), 71-93. 
14. ENCHÉRY, G. Modèles et schémas numériques pour la simulation de génèse de bassins sédimentaires. Thèse (2004).

15. Enchéry, G., Eymard, R., \& Michel, A. Numerical approximation of a two-phase flow problem in a porous medium with discontinuous capillary forces. SIAM J. Numer. Anal. 43 (2006), 2402-2422. Zbl 1145.76046 MR 2206441

16. Ersland, B. G., Espedal, M. S. \& Nybo, R. Numerical methods for flows in a porous medium with internal boundary. Comput. Geosci. 2 (1998), 217-240. Zbl 0943.76059 MR 1690664

17. Eymard, R., Gallouët, T., \& Herbin, R. Finite volume methods. Handbook of Numerical Analysis, Vol. 7, P. G. Ciarlet and J.-L. Lions (eds.), North-Holland (2000), 713-1020. Zbl 0981.65095 MR 1804748

18. Friedman, A. Partial Differential Equations of Parabolic Type. Prentice-Hall, Englewood Cliffs, NJ (1964). Zbl 0144.34903 MR 0181836

19. Gagneux, G., \& Madaune-Tort, M. Analyse mathématique de modèles non linéaires de l'ingénierie pétrolière. Math. Appl. 22, Springer, Berlin (1996). Zbl 0842.35126 MR 1616513

20. KARlsen, K. H., Risebro, N. H., \& Towers, J. D. On a nonlinear degenerate parabolic transportdiffusion equation with a discontinuous coefficient. Electron. J. Differential Equations 2002, no. 93, 23 pp. Zbl 1015.35049 MR 1938389

21. Karlsen, K. H., Risebro, N. H., \& Towers, J. D. Upwind difference approximations for degenerate parabolic convection-diffusion equations with a discontinuous coefficient. IMA J. Numer. Anal. 22 (2002), 623-664. Zbl 1014.65073 MR 1937244

22. Karlsen, K. H., Risebro, N. H., \& Towers, J. D. $L^{1}$ stability for entropy solutions of nonlinear degenerate parabolic convection-diffusion equations with discontinuous coefficients. Skr. K. Nor. Vidensk. Selsk. 2003, no. 3, 1-49. Zbl 1036.35104 MR 2024741

23. KRUŽKOV, S. N. First order quasilinear equations in several independent variables. Math. USSR-Sb. 81 (1970), 217-243.

24. Ladyzhenskaya, O. A., Solonnikov, V. A., \& Ural'Ceva, N. N. Linear and Quasi-Linear Equations of Parabolic Type. Transl. Math. Monogr. 23, Amer. Math. Soc., Providence, RI (1968). Zbl 0174.15403 MR 0241822

25. LERAY, J., \& LiONS, J.-L. Quelques résultats de Višik sur les problèmes elliptiques non linéaires par les méthodes de Minty-Browder. Bull. Soc. Math. France 93 (1965), 97-107. Zbl 0132.10502 |MR 0194733

26. MÁlek, J., NeČAs, J., Rokyta, M., \& RŮŽIČKA, M. Weak and Measure-Valued Solutions to Evolutionary PDEs. Chapman \& Hall, London (1996). Zbl 0851.35002 MR 1409366

27. Отто, F. $L^{1}$-contraction and uniqueness for quasilinear elliptic-parabolic equations. J. Differential Equations 131 (1996), 20-38. Z Zbl 0862.35078 MR 1415045 